\title{
$\mathrm{M}|\mathrm{R}| \mathrm{S}$ Internet Journal Nitride Semiconductor Research
}

\section{High Temperature Elastic Constant Prediction of Some Group III- Nitrides}

\author{
Robert R. Reeber ${ }^{1}$ and Kai Wang ${ }^{1}$ \\ ${ }^{1}$ Department of Materials Science and Engineering, North Carolina State University,
}

(Received Tuesday, January 9, 2001; accepted Thursday, January 25, 2001)

Thermoelastic properties are important for modeling thermal residual stresses and for optimizing the growth conditions of semiconductor thin films. Thermal expansions of AlN and GaN have been evaluated and predicted by us earlier [1] [2]. Here, high temperature elastic constants are estimated empirically from corresponding state relationships and data from other hexagonal Grimm-

Sommerfeld compounds. This information together with our earlier thermal expansion data will further improve capabilities for calculating thermal residual stresses in various semiconductor thin films.

\section{Introduction and Methods}

A material at absolute zero is uniquely represented by the nature of its atoms, their spatial arrangement and the forces between them. In the rigid ion model it is assumed that the electron distribution is locked to the nuclei and is not affected by the lattice dynamics induced by increases in temperature and pressure. As one explores the state of matter at higher temperatures and pressures it is anticipated that most models will have increasing inaccuracies as interatomic interactions responsible for materials behavior are complex, generally non-linear, and are not well understood. Shell representations provide qualitative results for this difficult problem. First principle theories for calculating high temperature elastic constants have many approximations and assumptions and, as indicated earlier [3] [4], have difficulty in reproducing thermal expansion measurements over extended temperature ranges. Calculations of high temperature elastic constants [5] based upon the central force model of Keating [6] [7] also have been questioned on theoretical grounds [8]. Most of the theoretical work provides zero $\mathrm{K}$ results for the temperature dependence of assumed quasi-harmonic cubic semiconductors.

It is well known that the thermophysical properties of crystal-chemically similar materials often can be simply correlated by corresponding states relationships [9]. The symmetry of such materials provides a specific mathematical framework for describing the distribution of the fully excited $3 \mathrm{~N}$ frequencies of a mole of atoms or molecules of a specific crystal structure. The stronger the material the higher is the temperature range that equivalent vibrational modes become excited. Simple correlations for melting, phase transitions and brittle to ductile transition temperatures have been made earlier [10] [11] [12].

In this work we use Debye temperatures as empirical corresponding states parameters to determine the elastic constants of $\mathrm{SiC}, \mathrm{AlN}, \mathrm{GaN}$ and $\mathrm{InN}$ from measured elastic constants of CdS [13]. It is assumed that:

$$
\frac{C_{i j}^{A}\left(T^{A}\right)}{C_{i j}^{A}\left(\theta_{D}^{A}\right)}=\frac{C_{i j}^{B}\left(T^{B}\right)}{C_{i j}^{B}\left(\theta_{D}^{B}\right)}
$$

Where:

$$
T^{A}=T^{B} \theta_{D}^{A} / \theta_{D}^{B}
$$

for compounds A and B. Li and Bradt [14] have predicted the high temperature elastic constants for $6 \mathrm{H} \alpha$ silicon carbide. Their results are utilized as a test for our predictions.

\section{Results}

Table 1 lists the experimental literature values of the elastic constants for $\mathrm{SiC}, \mathrm{GaN}, \mathrm{AlN}$ and $\mathrm{InN}$ at room temperature. Elastic constants of $\mathrm{SiC}$ as measured by several authors [15] [16] [17] are also given in Table 1. Table 2 provides our predictions for $6 \mathrm{H}-\mathrm{SiC}$. With the exception of a $13 \%$ difference for $\mathrm{C}_{13}$ the relative differ- 
ences are significantly less than $7 \%$ with respect to $\mathrm{Li}$ and Bradt's [14] results. The calculated Young's modulus utilizing the Voigt-Ruess-Hill (VRH) average is within $-2.3 \%$ at room temperature to $+0.27 \%$ at $1250^{\circ} \mathrm{K}$ of Li and Bradt's results. Tables 3, 4 and 5 provide our predictions for AlN, GaN and InN. The room temperature elastic constant values necessary for our calculations are based upon literature results [18] [19] [20] [21] [22] [23] [24] [25] [26] [27] [28]. The Young's moduli calculated from McNeil et al.'s data [19] for AlN are shown in Figure 1 with existing experimental data [18] [19] [20] [29] [30] [31] [32] [33] [34] [35] [36]. For $\mathrm{GaN}$, the room temperature elastic constants are based on the average of consistent measurements [20] [22] [24] [26] [27]. For InN, theoretical values from Wright [37] are applied in our calculation. Although experimental data exists for $\mathrm{InN}, \mathrm{GaN}$ results generated by the same authors are not consistent with others [28]. The temperature dependence of the Young's moduli for other compounds investigated are provided in Figure 2.

\section{Discussion and Conclusions}

A complete set of the elastic constants for important semiconducting nitrides has been calculated over an extended temperature range by utilizing an empirical corresponding state relationship. Values shown for the experimentally measured Young's moduli of AlN of Lubis et al. [30] and Ruh et al. [33] are in reasonable agreement with our calculations. They had some porosity in their polycrystalline samples. At higher temperatures one might expect a brittle to ductile transition to occur. This would cause a reduction in the measured Young's modulus. Unfortunately the mechanical property data available for comparison with our calculations is too limited to determine if such a transition occurs. In any case our results should be useful for estimating relative thermal residual stress variations under different growth conditions.

\section{ACKNOWLEDGMENTS}

The author appreciated the support of the Army Research Office through Grant No. DAAH04-93-D0003. This work was carried out while one of the authors (K.W.) held a National Research CouncilARO Research Associateship.

\section{REFERENCES}

[1] K. Wang, R. R. Reeber, Mater. Res. Soc. Symp. Proc. 482, 863 (1998).

[2] R. R. Reeber, K. Wang, J. Mater. Res. 15, 40 (2000).

[3] R. R. Reeber, K. Wang, J. Electron. Mater. 25, 63 (1996).

[4] R. R. Reeber, K. Wang, Mater. Chem. Phys. 46, 259 (1996).
[5] A. I. Gubanov, S. Y. Davydov, Sov. Phys. Solid State 14, 372 (1972).

[6] P. N. Keating, Phys. Rev. 145, 637 (1966).

[7] P. N. Keating, Phys. Rev. 149, 674 (1966).

[8] J. W. Martin, J. Phys. C 8, 2869 (1975).

[9] M. Blackman,"The specific heat of solids", Hdb. Phys. Vol. VII/I, 325 (1955)

[10] R. R. Reeber, Phys. Stat. Sol. A 26, 253 (1974).

[11] R. R. Reeber, "An empirical method for correlating and predicting melting and solid-state phase transformations", in The physics and chemistry of minerals and rocks, edited by $\mathrm{R}$. G. J. Strens (John Wiley, New York, 1976), p. 469

[12] R. R. Reeber, "Corresponding state aspects of the brittle to ductile transition in diamond and sphalerite-structure solids", in Fracture Mechanics of Ceramics; Vol. 6., edited by R. C. Bradt, A. G. Evans, D. P. H. Hasselman, and F. F. Lange (Plenum, 1983), p. 545

[13] D. Gerlich, J. Phys. Chem. Sol. 28, 2575 (1967).

[14] Z. Li, R. C. Bradt, Int. J. High Tech. Ceram. 4, 1 (1988).

[15] G. Arlt, G. R. Schodder, J. Acoust. Soc. of Am. 37, 384 (1965).

[16] K. Kamitani, M. Grimsditch, J. C. Nipko, C. -K. Loong, M. Okada, I. Kimura, J. Appl. Phys. 82, 3152 (1997).

[17] S. Karmann, R. Helbig, R. A. Stein, J. Appl. Phys. 66, 3922 (1989).

[18] K. Tsubouchi, K. Sugai, N. Mikoshiba, "AlN materials constants evaluation and SAW properties on $\mathrm{AlN} / \mathrm{Al}_{2} \mathrm{O}_{3}$ and AlN/Si", in 1981 Ultrasonics Symposium Proceedings, 1981 (IEEE. NewYork), p. 375

[19] LE McNeil, M Grimsditch, RH French, J. Am. Ceram. Soc. 76, 1132-1136 (1993).

[20] C. Deger, E. Born, H. Angerer, O. Ambacher, M. Stutzmann, J. Hornsteiner, E. Riha, G. Fischerauer, Appl. Phys. Lett. 72, 2400 (1998).

[21] V. A. Savastenko, A. U. Sheleg, Phys. Stat. Sol. A 48, K135 (1978).

[22] A. Polian, M. Grimsditch, I. Grzegory, J. Appl. Phys. 79, 3343-3344 (1996).

[23] Y. Takagi, M. Ahart, T. Azuhata, T. Sota, K. Suzuki, S. Nakamura, Physica B 219/220, 547 (1996).

[24] M. Yamaguchi, T. Yagi, T. Azuhata, T. Sota, K. Suzuki, S. Chichibu, S. Nakamura, J. Phys. C 9, 241 (1997).

[25] R. B. Schwarz, K. Khachaturyan, E. R. Weber, Appl. Phys. Lett. 70, 1122 (1997).

[26] T. Deguchi, D. Ichiryu, K. Toshikawa, K. Sekiguchi, T. Sota, R. Matsuo, T. Azuhata, T. Yagi, S. Chichibu, S. Nakamura, J. Appl. Phys. 86, 1860 (1999).

[27] M. Yamaguchi, T. Yagi, T. Sota, T. Deguchi, K. Shimada, S. Nakamura, J. Appl. Phys. 85, 8502 (1999).

[28] A. U. Sheleg, V. A. Savastenko, Inorg. Mat. 15, 1257 (1979).

[29] K. M. Taylor, C. Lenie, J. Electrochem. Soc. 107, 308 (1960).

[30] A. H. Lubis, N. L. Hecht, J. G. A. Graves, J. Am. Ceram. Soc. 82, 2481 (1999).

[31] D. Gerlich, S. L. Dole, G. A. Slack, J. Phys. Chem. Sol. 47, 437 (1986). 
[32] P. Boch, J. C. Glandus, J. Jarrige, J. P. Lecompte, J. Mexmain, Ceram. Int. 8, 34 (1982).

[33] R. Ruh, A. Zangvil, J. Barlowe, Am. Ceram. Soc. Bull. 64, 1368 (1985).

[34] A. Kampfe, B. Eigenmann, O. Vöhringer, D. Löhe, High Temp. Material Processes 2, 309 (1998).

[35] G. Carlotti, D. Fioretto, L. Giovannini, L. Palmieri, G. Socino, L. Verdini, E. Verona, "1994 IEEE Ultrasonic Symposium Proceedings", in 1994 IEEE Ultrasonic Symposium Proceedings, 1994 (IEEE New York), p. 457

[36] T. Shiosaki, T. Yamamoto, T. Oda, K. Harada, A. Kawabata, "Low temperature growth of piezoelectric AlN film for surface and bulk wave transducers by RF reactive planer magnetron sputtering", in 1980 IEEE Ultrasonic Symposium Proceedings, Boston, MA, 1980 (IEEE), p. 451

[37] AF Wright, J. Appl. Phys. 82, 2833-2839 (1997).

\section{FIGURES}

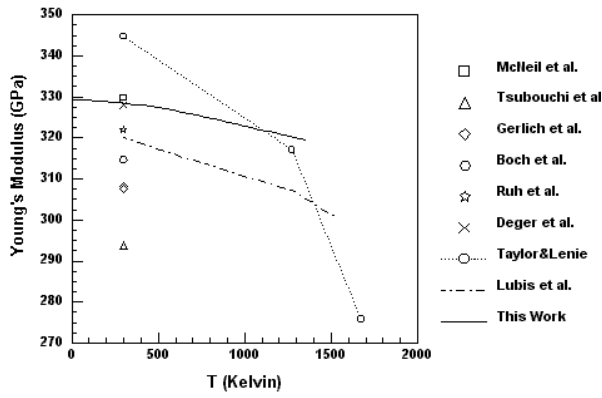

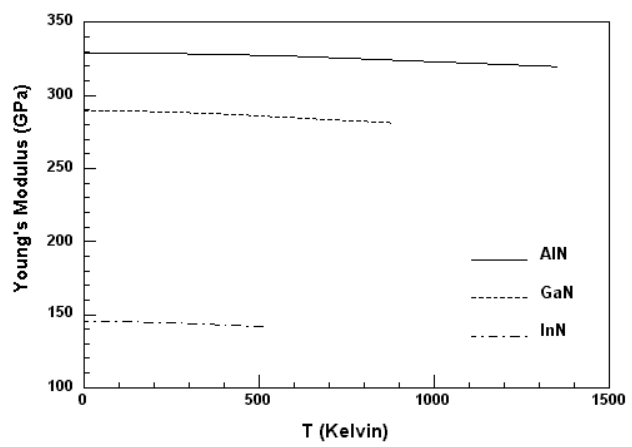

Figure 2. Temperature dependence of Young's Modulus for $\mathrm{AlN}, \mathrm{GaN}$ and InN.

\section{TABLES}

Figure 1. Young's Modulus of AlN

Table 1. Room temperature elastic constants and Debye temperatures for SiC, AlN, GaN and InN.

\begin{tabular}{|c|c|c|c|c|c|c|c|}
\hline Compound & $\mathrm{C}_{11}(\mathrm{GPa})$ & $\mathrm{C}_{12}(\mathrm{GPa})$ & $\mathrm{C}_{13}(\mathrm{GPa})$ & $\mathrm{C}_{33}(\mathrm{GPa})$ & $\mathrm{C}_{44}(\mathrm{GPa})$ & $\theta_{\mathrm{D}}(\mathrm{K})$ & Ref. \\
\hline \multirow[t]{9}{*}{ GaN } & 296.0 & 130.0 & 158.0 & 267.0 & 24.1 & & [21] \\
\hline & 390.0 & 145.0 & 106.0 & 398.0 & 105.0 & & [22] \\
\hline & 374.0 & 106.0 & 70.0 & 379.0 & 101.0 & & [23] \\
\hline & 365.0 & 135.0 & 114.0 & 381.0 & 109.0 & & [24] \\
\hline & 377.0 & 160.0 & 114.0 & 209.0 & 81.4 & & [25] \\
\hline & 370.0 & 145.0 & 110.0 & 390.0 & 90.0 & & {$[20]$} \\
\hline & 373.0 & 141.0 & 80.4 & 387.0 & 93.6 & & [26] \\
\hline & 373.0 & 141.0 & 80.0 & 387.0 & 94.0 & & [27] \\
\hline & 374.2 & 141.4 & 98.1 & 388.6 & 98.3 & 637.3 & * \\
\hline \multirow[t]{7}{*}{$A \perp N$} & & & & 404.0 & & & [36] \\
\hline & 345.0 & 125.0 & 120.0 & 395.0 & 118.0 & & [18] \\
\hline & 410.5 & 148.5 & 98.9 & 388.5 & 124.6 & & [19] \\
\hline & & & & & 102.0 & & [35] \\
\hline & 410.0 & 140.0 & 100.0 & 390.0 & 120.0 & & [20] \\
\hline & 424.0 & 122.0 & 166.0 & 353.0 & 123.0 & & [34] \\
\hline & 410.5 & 148.5 & 98.9 & 388.5 & 124.6 & 965.2 & * \\
\hline \multirow[t]{2}{*}{ InN } & 190.0 & 104.0 & 121.0 & 182.0 & 9.9 & & [28] \\
\hline & 223.0 & 115.0 & 92.0 & 224.0 & 48.0 & 375.5 & * \\
\hline \multirow[t]{5}{*}{ SiC } & 500.0 & 92.0 & & 564.0 & 168.0 & & [15] \\
\hline & 504.0 & 98.0 & & 566.0 & 170.0 & & [15] \\
\hline & & & & 564.9 & & & [17] \\
\hline & 501.0 & 111.0 & 52.0 & 553.0 & 163.0 & & [16] \\
\hline & 479.3 & 98.1 & 55.8 & 521.6 & 148.3 & 1123.8 & * \\
\hline $\begin{array}{l}\text { * Recomme } \\
\text { values }\end{array}$ & & & & & & & \\
\hline
\end{tabular}


Table 2. Elastic Properties of $6 \mathrm{H}-\mathrm{SiC}$

\begin{tabular}{|c|c|c|c|c|c|c|}
\hline $\mathrm{T} \quad(\mathrm{K})$ & $\mathrm{C}_{11}(\mathrm{GPa})$ & $\mathrm{C}_{33}(\mathrm{GPa})$ & $\mathrm{C}_{12}(\mathrm{GPa})$ & $\mathrm{C}_{13}(\mathrm{GPa})$ & $\mathrm{C}_{44}(\mathrm{GPa})$ & $E(\mathrm{GPa})$ \\
\hline 0 & 469.3 & 514.3 & 91.5 & 48.3 & 143.8 & 410.8 \\
\hline 50 & 469.3 & 514.4 & 91.5 & 48.3 & 143.8 & 410.8 \\
\hline 100 & 469.3 & 514.3 & 91.5 & 48.3 & 143.8 & 410.8 \\
\hline 150 & 469.1 & 514.1 & 91.4 & 48.2 & 143.7 & 410.7 \\
\hline 200 & 468.9 & 513.7 & 91.4 & 48.2 & 143.7 & 410.6 \\
\hline 250 & 468.6 & 513.5 & 91.3 & 48.2 & 143.7 & 410.4 \\
\hline 300 & 468.3 & 513.1 & 91.3 & 48.1 & 143.7 & 410.1 \\
\hline 400 & 467.6 & 512.2 & 91.1 & 48.0 & 143.6 & 409.6 \\
\hline 500 & 466.8 & 511.1 & 90.9 & 47.8 & 143.5 & 409.0 \\
\hline 600 & 465.8 & 509.5 & 90.6 & 47.7 & 143.3 & 408.2 \\
\hline 700 & 464.7 & 508.0 & 90.4 & 47.5 & 143.2 & 407.4 \\
\hline 800 & 463.4 & 506.3 & 90.1 & 47.3 & 143.0 & 406.4 \\
\hline 900 & 461.9 & 504.6 & 89.7 & 47.1 & 142.8 & 405.3 \\
\hline 1000 & 460.4 & 502.9 & 89.4 & 46.9 & 142.6 & 404.2 \\
\hline 1100 & 458.9 & 501.2 & 89.0 & 46.7 & 142.5 & 403.2 \\
\hline 1200 & 457.2 & 499.6 & 88.6 & 46.5 & 142.3 & 402.1 \\
\hline 1300 & 455.6 & 497.9 & 88.2 & 46.3 & 142.1 & 401.0 \\
\hline 1400 & 454.1 & 496.2 & 87.9 & 46.1 & 141.9 & 399.9 \\
\hline 1500 & 452.5 & 494.5 & 87.5 & 45.8 & 141.7 & 398.8 \\
\hline 1550 & 451.8 & 493.6 & 87.3 & 45.7 & 141.7 & 398.3 \\
\hline
\end{tabular}

Table 3. Elastic Properties of AlN

\begin{tabular}{|c|c|c|c|c|c|c|}
\hline $\mathrm{T}(\mathrm{K})$ & $\mathrm{C}_{11}(\mathrm{GPa})$ & $\mathrm{C}_{33}(\mathrm{GPa})$ & $\mathrm{C}_{12}(\mathrm{GPa})$ & $\mathrm{C}_{13}(\mathrm{GPa})$ & $\mathrm{C}_{44}(\mathrm{GPa})$ & $E(\mathrm{GPa})$ \\
\hline 0 & 411.6 & 385.5 & 148.9 & 99.4 & 124.1 & 329.2 \\
\hline 50 & 411.6 & 385.6 & 148.9 & 99.4 & 124.1 & 329.2 \\
\hline 100 & 411.5 & 385.5 & 148.9 & 99.3 & 124.1 & 329.2 \\
\hline 150 & 411.3 & 385.2 & 148.8 & 99.3 & 124.1 & 329.0 \\
\hline 200 & 411.1 & 385.0 & 148.7 & 99.2 & 124.1 & 328.9 \\
\hline 250 & 410.7 & 384.7 & 148.6 & 99.0 & 124.0 & 328.7 \\
\hline 300 & 410.5 & 384.3 & 148.4 & 98.9 & 124.0 & 328.5 \\
\hline 400 & 409.7 & 383.4 & 148.1 & 98.5 & 123.9 & 328.0 \\
\hline 500 & 408.7 & 382.1 & 147.6 & 98.1 & 123.7 & 327.4 \\
\hline 600 & 407.5 & 380.8 & 147.1 & 97.7 & 123.6 & 326.6 \\
\hline 700 & 406.2 & 379.4 & 146.6 & 97.2 & 123.4 & 325.8 \\
\hline 800 & 404.7 & 377.9 & 145.9 & 96.7 & 123.2 & 324.8 \\
\hline 900 & 403.1 & 376.4 & 145.2 & 96.2 & 123.0 & 323.9 \\
\hline 1000 & 401.5 & 374.9 & 144.5 & 95.7 & 122.9 & 322.9 \\
\hline 1100 & 399.9 & 373.4 & 143.8 & 95.3 & 122.7 & 321.9 \\
\hline 1200 & 398.3 & 372.0 & 143.1 & 94.8 & 122.5 & 321.0 \\
\hline 1300 & 396.7 & 370.5 & 142.4 & 94.3 & 122.3 & 320.0 \\
\hline 1350 & 396.0 & 369.8 & 141.9 & 94.0 & 122.3 & 319.6 \\
\hline
\end{tabular}


Table 4. Elastic Properties of GaN

\begin{tabular}{|c|c|c|c|c|c|c|}
\hline $\mathrm{T} \quad(\mathrm{K})$ & $\mathrm{C}_{11}(\mathrm{GPa})$ & $\mathrm{C}_{33}(\mathrm{GPa})$ & $\mathrm{C}_{12}(\mathrm{GPa})$ & $\mathrm{C}_{13}(\mathrm{GPa})$ & $\mathrm{C}_{44}(\mathrm{GPa})$ & $\mathrm{E}(\mathrm{GPa})$ \\
\hline 0 & 376.4 & 387.1 & 142.4 & 99.1 & 98.5 & 289.5 \\
\hline 50 & 376.4 & 387.1 & 142.4 & 99.1 & 98.5 & 289.5 \\
\hline 100 & 376.1 & 386.8 & 142.3 & 99.0 & 98.5 & 289.3 \\
\hline 150 & 375.7 & 386.5 & 142.1 & 98.9 & 98.5 & 289.1 \\
\hline 200 & 375.4 & 385.8 & 141.9 & 98.7 & 98.4 & 288.9 \\
\hline 250 & 374.8 & 385.2 & 141.7 & 98.4 & 98.4 & 288.5 \\
\hline 300 & 374.2 & 384.4 & 141.4 & 98.1 & 98.3 & 288.1 \\
\hline 400 & 372.6 & 382.3 & 140.6 & 97.4 & 98.1 & 287.2 \\
\hline 500 & 370.7 & 380.0 & 139.8 & 96.7 & 97.9 & 286.0 \\
\hline 600 & 368.5 & 377.8 & 138.8 & 96.0 & 97.7 & 284.8 \\
\hline 700 & 366.3 & 375.5 & 137.7 & 95.3 & 97.5 & 283.5 \\
\hline 800 & 364.1 & 373.3 & 136.7 & 94.5 & 97.3 & 282.3 \\
\hline 900 & 362.0 & 371.1 & 135.6 & 93.8 & 97.1 & 281.1 \\
\hline
\end{tabular}

Table 5. Elastic Properties of InN

\begin{tabular}{|c|c|c|c|c|c|c|}
\hline $\mathrm{T} \quad(\mathrm{K})$ & $\mathrm{C}_{11}(\mathrm{GPa})$ & $\mathrm{C}_{33}(\mathrm{GPa})$ & $\mathrm{C}_{12}(\mathrm{GPa})$ & $\mathrm{C}_{13}(\mathrm{GPa})$ & $\mathrm{C}_{44}(\mathrm{GPa})$ & $E(\mathrm{GPa})$ \\
\hline 0 & 226.7 & 225.8 & 117.2 & 94.4 & 48.3 & 145.5 \\
\hline 50 & 226.6 & 225.7 & 117.2 & 94.3 & 48.3 & 145.4 \\
\hline 100 & 226.2 & 225.3 & 116.9 & 94.1 & 48.3 & 145.3 \\
\hline 150 & 225.7 & 224.6 & 116.6 & 93.6 & 48.2 & 145.1 \\
\hline 200 & 225.0 & 223.7 & 116.1 & 93.1 & 48.2 & 144.7 \\
\hline 250 & 224.1 & 222.7 & 115.6 & 92.6 & 48.1 & 144.3 \\
\hline 300 & 223.1 & 221.6 & 115.0 & 92.0 & 48.0 & 143.9 \\
\hline 350 & 222.0 & 220.5 & 114.3 & 91.4 & 47.9 & 143.4 \\
\hline 400 & 220.9 & 219.4 & 113.5 & 90.8 & 47.8 & 142.9 \\
\hline 450 & 219.7 & 218.2 & 112.8 & 90.2 & 47.7 & 142.4 \\
\hline 500 & 218.6 & 217.1 & 112.1 & 89.6 & 47.6 & 141.9 \\
\hline
\end{tabular}

